\title{
Echoes in brane worlds: ringing at a black hole--wormhole transition
}

\author{
Kirill A. Bronnikov ${ }^{1,2,3}$ and Roman A. Konoplya ${ }^{4,2, *}$ \\ ${ }^{1}$ VNIIMS, Ozyornaya ul. 46, Moscow 119361, Russia \\ ${ }^{2}$ Inst. of Gravitation and Cosmology, RUDN University, \\ ul. Miklukho-Maklaya 6, Moscow 117198, Russia \\ ${ }^{3}$ National Research Nuclear University "MEPhI", Kashirskoe sh. 31, Moscow 115409, Russid $\dagger^{\dagger}$ \\ ${ }^{4}$ Institute of Physics and Research Centre of Theoretical Physics and Astrophysics, \\ Faculty of Philosophy and Science, Silesian University in Opava, CZ-746 01 Opava, Czech Republic
}

\begin{abstract}
Echoes are known as modifications of the usual quasinormal ringing of a black hole at late times because of the deviation of space-time from the initial black-hole geometry in a small region near its event horizon. We consider a class of brane-world model solutions of the Shiromizu-Maeda-Sasaki equations, which describe both black holes and wormholes and interpolate between them via a continuous parameter. In this way the brane-world scenario provides a natural model for wormholes mimicking the black hole behavior if the continuous parameter is chosen near the threshold with a black-hole solution. We show that in the vicinity of this threshold interpolating between black holes and wormholes, quasinormal ringing of the wormholes at the initial stage is indistinguishable from that of the black holes with nearby values of the above parameter, but at later times the signal is modified by intensive echoes. We notice that the black-hole mimickers that are wormholes near the threshold have the largest quality factor and are therefore the best oscillators among the considered examples.
\end{abstract}

\section{INTRODUCTION}

Recent observations of black holes (BHs) and stars in the electromagnetic and gravitational channels [1-4 allow us to test the regime of strong gravity. Nevertheless, nowadays there is still large uncertainty in measuring the parameters of compact objects, such as their mass and angular momentum, which leaves considerable freedom for various interpretations of the geometries either towards BHs in modified theories of gravity or even in favor of such exotic objects as stable Schwarzschild stars or wormholes [5]11.

A wormhole geometry can be designed ad hoc in such a way that it would mimic the behavior of a $\mathrm{BH}$ in any astrophysically relevant processes (except for the Hawking radiation which is unlikely to be observed for large $\mathrm{BHs}$ ) [5. This is possible because the geometry of such a wormhole can be indistinguishable from that of the $\mathrm{BH}$ in the whole space outside a tiny region near the wormhole throat. At intermediately late times, classical radiation in the vicinity of a $\mathrm{BH}$ or wormhole must be dominated by the characteristic damped oscillations, quasinormal modes, which have been extensively studied [12 14 and were recently observed in, apparently, mergers of two BHs [1, 2].

While quasinormal modes of wormholes mimicking $\mathrm{BHs}$ are almost the same, the signal is modified at later times by echoes, what was first observed in 11 and further studied in a large number of papers (see, for example, 16 29 and references therein). In [11, 16 the echoes produced by various toy models of thin-shell wormholes were studied. A similar picture of echoes at the beginning

* $\frac{\text { roman.konoplya@gmail.com }}{\text { kb20@yandex.ru }}$ of the threshold between a regular $\mathrm{BH}$ and a wormhole has been found in 15 for the metric suggested in 30. There, a continuous parameter of the metric allowed for interpolation between a regular $\mathrm{BH}$, a one-way wormhole with an extremal null throat (the so called black bounce) and a traversable wormhole. However, the above wormhole metric is not a solution of any field equations and was simply designed ad hoc in the same manner as the Damour-Solodukhin wormhole [5] whose echoes were studied in 11 .

A few examples of families of exact solutions containing both BHs and wormholes have been found with phantom scalar fields as sources of gravity (see, e.g., 31 34]), however, such solutions have been shown to be generically unstable under radial perturbations 34 37.

Here we will also consider various geometries interpolating between $\mathrm{BHs}$ and traversable wormholes via a continuous parameter, but which, unlike the previous models, are exact solutions of the Shiromizu-MaedaSasaki equations 38, describing the on-brane gravitational field in the second Randall-Sundrum brane-world scenario (RS2) 39]. The exact solutions to be studied concerning the possible echoes and quasinormal modes were obtained in 40, 41, and quasinormal modes in the frequency and time domains were studied in [44, but only for the range of parameters representing BHs. Thus the effect of echoes which should take place for wormholes in the parameter range near the threshold with BHs was missed in 44 .

Having all the above motivations in mind, we would like to consider the quasinormal modes and ringing profiles for a few examples of the $\mathrm{BH}$ /wormhole solutions of the Shiromizu-Maeda-Sasaki equations in order to understand the possible general imprints of this extradimensional scenario on the ringing profile of $\mathrm{BHs}$ and wormholes, and especially at the transition between 
them. We will show that once the continuous parameter interpolating between the $\mathrm{BH}$ and wormhole solutions describes a wormhole near the "transition," the quasinormal ringing is represented by damping oscillations appropriate for the near-threshold $\mathrm{BH}$ solution, but modified by echoes at later times. When the continuous parameter is further increased, then the echoes go over into the characteristic quasinormal ringing of the wormhole, while the period of the initial "threshold BH phase" damped oscillations diminishes and looks more like an initial outburst. The term "transition" must certainly be understood here with a considerable reservation: strictly speaking, we can only talk about values of this parameter making the geometry of a wormhole better or worse $\mathrm{BH}$ mimicker. Nevertheless, if one supposes that this parameter adiabatically changes with time as a result of some brane-world dynamic process, then the time-domain profiles of perturbations which relax at a much higher rate than the adiabatic change of the metric are qualitatively the same, as was shown, for example, in 61.

The paper is organized as follows. In Sec. II we briefly summarize the basic information about the brane-world model under consideration. Sec. III discusses the wave equations for test scalar and electromagnetic fields and the WKB and time-domain integration methods to be used for the analysis of ringing. Sec. IV is devoted to the quasinormal ringing of a few examples of $\mathrm{BH}$ and wormhole solutions with special emphasis to the parametric range near the transition between them. Finally, in the Conclusions we summarize the obtained results.

\section{THE BRANE-WORLD MODEL}

We will consider the second Randall-Sundrum braneworld model (RS2) 39] implying that our fourdimensional world is a hypersurface supporting all matter fields and embedded in a $\mathbb{Z}_{2}$-symmetric five-dimensional spacetime (asymptotically AdS bulk), while the gravitational field propagates in the whole bulk. The gravitational field on the brane itself is described by the modified Einstein equations derived by Shiromizu, Maeda and Sasaki 38.

$$
G_{\mu}^{\nu}=-\Lambda_{4} \delta_{\mu}^{\nu}-\kappa_{4}^{2} T_{\mu}^{\nu}-\kappa_{5}^{4} \Pi_{\mu}^{\nu}-E_{\mu}^{\nu},
$$

where $G_{\mu}^{\nu}=R_{\mu}^{\nu}-\frac{1}{2} \delta_{\mu}^{\nu} R$ is the $4 \mathrm{D}$ Einstein tensor, $\Lambda_{4}$ is the $4 \mathrm{D}$ cosmological constant expressed in terms of the $5 \mathrm{D}$ cosmological constant $\Lambda_{5}$ and the brane tension $\lambda$ :

$$
\Lambda_{4}=\frac{1}{2} \kappa_{5}^{2}\left(\Lambda_{5}+\frac{1}{6} \kappa_{5}^{2} \lambda^{2}\right)
$$

$\kappa_{4}^{2}=8 \pi G_{N}=\kappa_{5}^{4} \lambda /(6 \pi)$ is the $4 \mathrm{D}$ gravitational constant ( $G_{N}$ is the Newtonian constant of gravity); $T_{\mu}^{\nu}$ is the stress-energy tensor of matter located on the brane; $\Pi_{\mu}^{\nu}$ is a tensor quadratic in $T_{\mu}^{\nu}$, obtained from the matching conditions for the $5 \mathrm{D}$ metric across the brane:

$$
\Pi_{\mu}^{\nu}=\frac{1}{4} T_{\mu}^{\alpha} T_{\alpha}^{\nu}-\frac{1}{2} T T_{\mu}^{\nu}-\frac{1}{8} \delta_{\mu}^{\nu}\left(T_{\alpha \beta} T^{\alpha \beta}-\frac{1}{3} T^{2}\right)
$$

where $T=T_{\alpha}^{\alpha}$; lastly, $E_{\mu}^{\nu}$ is the so-called "electric" part of the $5 \mathrm{D}$ Weyl tensor projected onto the brane: in proper $5 \mathrm{D}$ coordinates, we have $E_{\mu \nu}=\delta_{\mu}^{A} \delta_{\nu}^{C(5)} C_{A B C D} n^{B} n^{D}$, where the capital letters $A, B, \ldots$ are $5 \mathrm{D}$ indices, and $n^{A}$ is the unit normal vector to the brane. By construction, $E_{\mu}^{\nu}$ is traceless, that is, $E_{\mu}^{\mu}=0$ [38. The general class and a number of particular examples describing wormholes and $\mathrm{BHs}$ in the above brane-world scenario were obtained in [40, 41].

A feature of utmost interest in these models is the generic appearance of families of solutions that unify symmetric wormholes and globally regular $\mathrm{BHs}$ with a minimum of the variable $r$ (bounce) in their T-regions and a Kerr-like global structure. These two qualitatively different parts of any such family are separated by an extremal $\mathrm{BH}$ solution.

In what follows we will consider different examples of such solutions: one family representing the generic situation, another one with only an extremal $\mathrm{BH}$, and the third one with a zero Schwarzschild mass. In each of these examples the metric is a vacuum solutions to Eqs. (1), with $T_{\mu}^{\nu}=0$ and $\Lambda_{4}=0$; the effective energy-momentum tensor in the r.h.s. of (1) is thus the "tidal" tensor $E_{\mu}^{\nu}$ of bulk origin. All metrics under consideration are asymptotically flat and have a zero Ricci scalar.

\section{THE METHODS}

Quasinormal modes of BHs are proper oscillations of BHs under specific boundary conditions, corresponding to purely outgoing waves at infinity and purely incoming waves at the event horizon (minus infinity in terms of the tortoise coordinate). The boundary conditions for a traversable wormhole which connects two infinities are the same in terms of the tortoise coordinate [45], so that many of the tools used for finding $\mathrm{BH}$ quasinormal modescan, with small modifications, be used for wormholes as well [37, 46, 47, 59, 60, 62, 63. In particular, to finding the frequencies of quasinormal modes, we will use the WKB method when the effective potential has a single maximum and time domain integration for all types of the effective potentials.

\section{A. Wave equations}

The metric of a spherically symmetric static space-time can be written in the general form ${ }^{1}$

$$
d s^{2}=-A(r) d t^{2}+B(r) d r^{2}+r^{2}\left(\sin ^{2} \theta d \phi^{2}+d \theta^{2}\right) .
$$

\footnotetext{
1 Note that we are using notations different from those in 41: $B(r)$ of the present paper is equal to $1 / B(r)$ of 41 .
} 
The generally covariant equation for a massless scalar field has the form

$$
\frac{1}{\sqrt{-g}} \partial_{\mu}\left(\sqrt{-g} g^{\mu \nu} \partial_{\nu} \Phi\right)=0
$$

and for an electromagnetic field

$$
\frac{1}{\sqrt{-g}} \partial_{\mu}\left(F_{\rho \sigma} g^{\rho \nu} g^{\sigma \mu} \sqrt{-g}\right)=0,
$$

where $F_{\rho \sigma}=\partial_{\rho} A_{\sigma}-\partial_{\sigma} A_{\rho}$ and $A_{\mu}$ is the vector potential. After separation of variables, Eqs. (5) and (6) take the following general Schrödinger-like form:

$$
\frac{d^{2} \Psi_{s}}{d r_{*}^{2}}+\left(\omega^{2}-V(r)\right) \Psi_{s}=0,
$$

where $s=0$ corresponds to the scalar field and $s=1$ to the electromagnetic field, the "tortoise coordinate" $r_{*}$ is defined by the relation

$$
d r_{*}=d r \sqrt{\frac{B(r)}{A(r)}}
$$

and the effective potentials are (see, e.g., Eq. (12a) in 54])

$$
\begin{aligned}
& V_{s}(r)=A(r) \frac{\ell(\ell+1)}{r^{2}}+\frac{1}{2 r} \frac{d}{d r} \frac{A(r)}{B(r)}, \\
& V_{e m}(r)=A(r) \frac{\ell(\ell+1)}{r^{2}} .
\end{aligned}
$$

\section{B. The WKB approach}

For the analysis in the frequency domain we shall use the semi-analytical WKB method 48 52. The essence of this approach is the expansion of the solution at both infinities in WKB series and matching these asymptotic expansions with the Taylor expansion near the peak of the effective potential. In addition, according to [50], we use a further representation of the WKB expansion in the form of the Padé approximants which, in most cases, greatly improves the accuracy of the WKB method. The WKB formula can be written in the following form [51]:

$$
\begin{aligned}
& \omega^{2}=V_{0}+A_{2}\left(\mathcal{K}^{2}\right)+A_{4}\left(\mathcal{K}^{2}\right)+A_{6}\left(\mathcal{K}^{2}\right)+\ldots \\
& -i \mathcal{K} \sqrt{-2 V_{2}}\left(1+A_{3}\left(\mathcal{K}^{2}\right)+A_{5}\left(\mathcal{K}^{2}\right)+A_{7}\left(\mathcal{K}^{2}\right) \ldots\right),
\end{aligned}
$$

where $\mathcal{K}=n+1 / 2, n=0,1,2,3 \ldots$

The corrections $A_{k}\left(\mathcal{K}^{2}\right)$ of order $k$ to the eikonal formula are polynomials in $\mathcal{K}^{2}$ with rational coefficients and depend on the values $V_{2}, V_{3} \ldots$ of higher-order derivatives of the potential $V(r)$ at its maximum. To increase accuracy of the WKB formula, we use the procedure suggested by Matyjasek and Opala [50, which consists in using the Padé approximants. For the order $k$ of the
WKB formula 11 we define a polynomial $P_{k}(\epsilon)$ in the following way

$$
\begin{aligned}
& P_{k}(\epsilon)=V_{0}+A_{2}\left(\mathcal{K}^{2}\right) \epsilon^{2}+A_{4}\left(\mathcal{K}^{2}\right) \epsilon^{4}+A_{6}\left(\mathcal{K}^{2}\right) \epsilon^{6}+\ldots \\
& -i \mathcal{K} \sqrt{-2 V_{2}}\left(\epsilon+A_{3}\left(\mathcal{K}^{2}\right) \epsilon^{3}+A_{5}\left(\mathcal{K}^{2}\right) \epsilon^{5} \ldots\right)
\end{aligned}
$$

and the squared frequency is obtained for $\epsilon=1$ :

$$
\omega^{2}=P_{k}(1)
$$

For the polynomial $P_{k}(\epsilon)$ we will use Padé approximants

$$
P_{\tilde{n} / \tilde{m}}(\epsilon)=\frac{Q_{0}+Q_{1} \epsilon+\ldots+Q_{\tilde{n}} \epsilon^{\tilde{n}}}{R_{0}+R_{1} \epsilon+\ldots+R_{\tilde{m}} \epsilon^{\tilde{m}}}
$$

with $\tilde{n}+\tilde{m}=k$, such that, near $\epsilon=0$,

$$
P_{\tilde{n} / \tilde{m}}(\epsilon)-P_{k}(\epsilon)=\mathcal{O}\left(\epsilon^{k+1}\right) \text {. }
$$

Usually, for finding the fundamental mode $(n=0)$ frequency, Padé approximants with $\tilde{n} \approx \tilde{m}$ provide the best approximation. In [50], $P_{6 / 6}(1)$ and $P_{6 / 7}(1)$ were compared to the 6th-order WKB formula $P_{6 / 0}(1)$. In [51] it has been observed that usually even $P_{3 / 3}(1)$, i. e. a Padé approximation of the 6 th-order gives a more accurate value for the squared frequency than $P_{6 / 0}(1)$. Here we will use the 7 th WKB expansions with $\tilde{m}=3$ Padé approximation and show that the results obtained at different WKB orders are in a very good agreement.

\section{Time-domain integration}

We will integrate the wavelike equations rewritten in terms of the light-cone variables $u=t-r_{*}$ and $v=t+r_{*}$. The appropriate discretization scheme was suggested in [53]:

$$
\begin{aligned}
& \Psi(N)=\Psi(W)+\Psi(E)-\Psi(S) \\
& -\Delta^{2} \frac{V(W) \Psi(W)+V(E) \Psi(E)}{8}+\mathcal{O}\left(\Delta^{4}\right)
\end{aligned}
$$

where we use the following designations for the points: $N=(u+\Delta, v+\Delta), W=(u+\Delta, v), E=(u, v+\Delta)$ and $S=(u, v)$. The initial data are specified on the null surfaces $u=u_{0}$ and $v=v_{0}$. To extract the values of the quasinormal frequencies, we will use the Prony method which allows us to fit the signal by a sum of exponentials with some excitation factors.

\section{THE PICTURE OF QUASINORMAL RINGING FOR VARIOUS SOLUTIONS}

\section{A. The Casadio-Fabbri-Mazzacurati (CFM) metric}

Our first example with the Schwarzschild generating function $A(r)=1-2 M / r$ [1] represnts the generic behavior of brane-world $\mathrm{BH} /$ wormhole families. The metric 

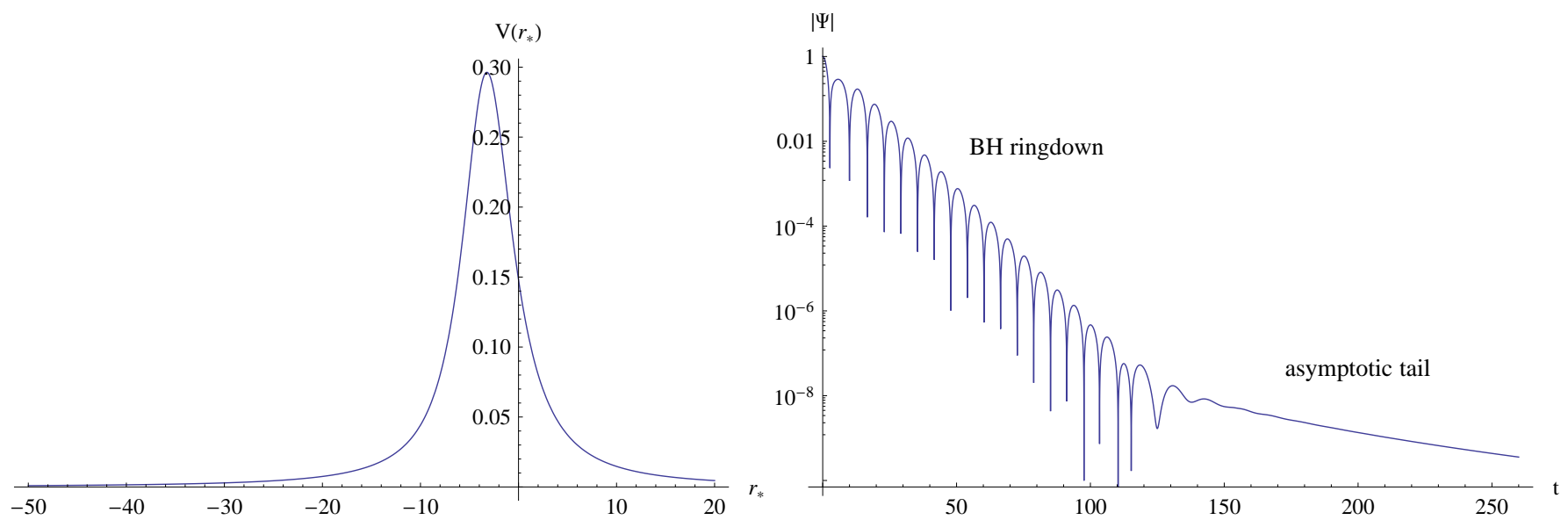

FIG. 1. The effective potential (left) and the semi-logarithmic plot of the time-domain profile (right) for perturbations of the electromagnetic field in the vicinity of the double horizon of CFM BHs with the metric 15 where $r_{0}=2 M, M=1 / 2, \ell=1$. The quasinormal ringing goes over into asymptotic power-law tails.
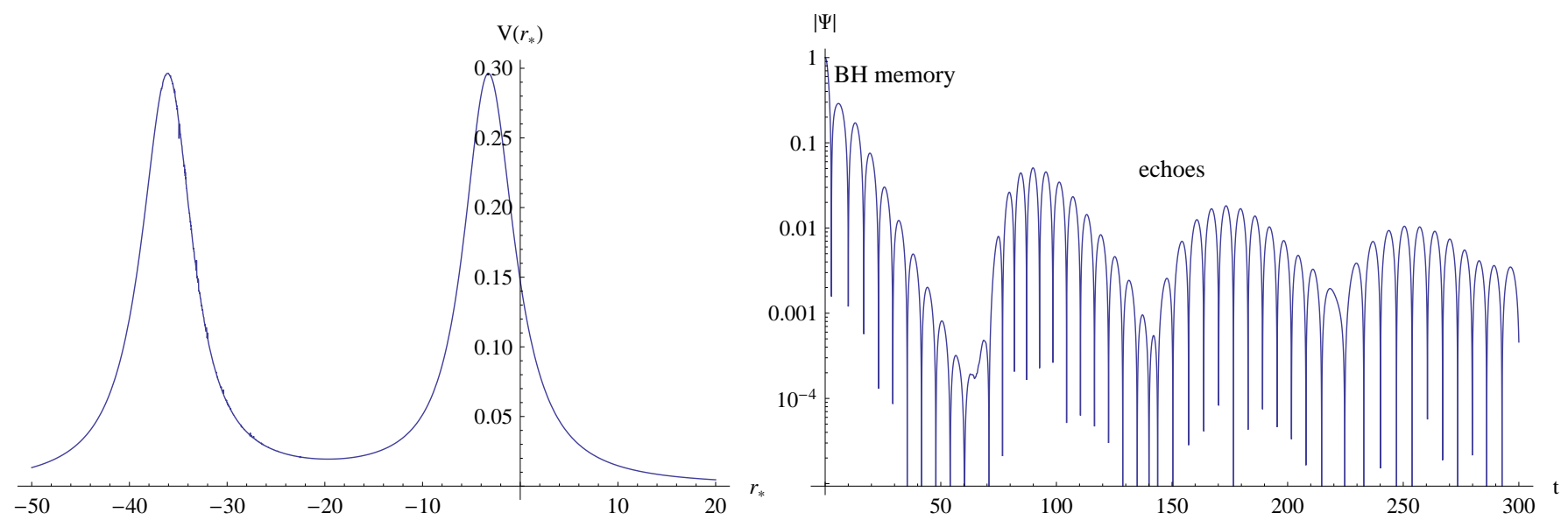

FIG. 2. The effective potential (left) and the semi-logarithmic plot of the time-domain profile (right) for perturbations of the electromagnetic field in the vicinity of a CFM wormhole with $M=1 / 2, r_{0}=1.01, \ell=1$. The first long period of damped oscillations is indistinguishable from that of the threshold $\mathrm{BH}$ metric (that corresponds to $r_{0}=2 M$ ), but, at later times it goes over into a series of echoes.

has the form

$$
\begin{aligned}
d s^{2} & =\left(1-\frac{2 M}{r}\right) d t^{2} \\
& -\frac{1-3 M /(2 r)}{(1-2 M / r)\left(1-r_{0} / r\right)} d r^{2}-r^{2} d \Omega^{2} .
\end{aligned}
$$

The Schwarzschild metric is reproduced in the special case $r_{0}=3 M / 2$. The metric (15) has been obtained by Casadio, Fabbri and Mazzacurati 42 in search for new brane-world BH solutions and by Germani and Maartens [43] as a possible metric outside a homogeneous star on the brane.

In the case $r_{0}>2 M$, the metric 15 describes a symmetric traversable wormhole [40.

In the intermediate case $r_{0}=2 M$ we obtain a $\mathrm{BH}$ with a double horizon at $r=2 M$.

In the case $r_{0}<2 M$, there is a BH with a sin- gle horizon at $r=2 M$. As described in Ref. 42, the global space-time structure depends on the sign of $\eta=r_{0}-3 M / 2$. If $\eta<0$, this structure coincides with that of a Schwarzschild $\mathrm{BH}$, but the spacelike curvature singularity is located at $r=3 M / 2$ instead of $r=0$. If $\eta>0$, the solution describes a nonsingular $\mathrm{BH}$ with a minimum value of the variable $r$ equal to $r_{0}>3 M / 2$ inside the horizon, that is, a bounce in the two angular directions of a Kantowski-Sachs anisotropic cosmology.

In Fig.1 one can see the usual picture of evolution of perturbations in the vicinity of a $\mathrm{BH}$, now for the threshold case $r_{0}=2 M$. It consists of the initial outburst which changes into damped quasinormal oscillations and powerlow asymptotic tails in the end. Figure 2 shows perturbations of a wormhole corresponding to $r_{0}=2.02 M$, that is, very close to the threshold with the $\mathrm{BH}$ state, and there emerges a distinctive picture of echoes after the initial os- 

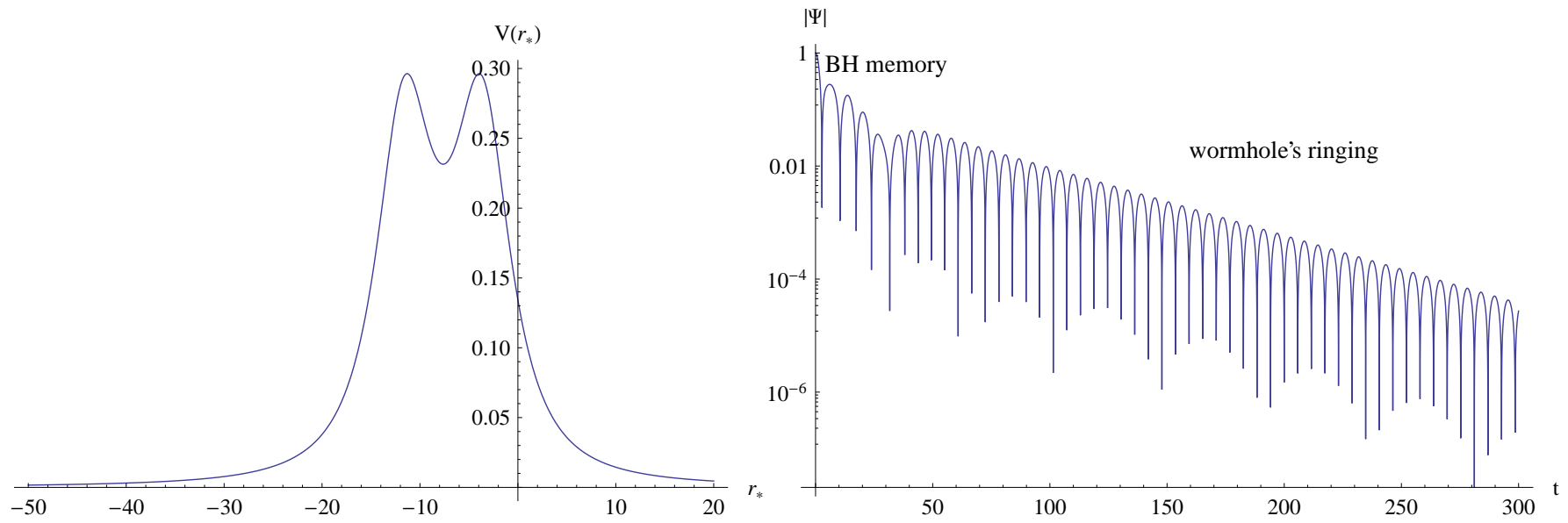

FIG. 3. The effective potential (left) and the semi-logarithmic plot of the time-domain profile (right) for perturbations of the electromagnetic field in the vicinity of a CFM wormhole with $M=1 / 2, r_{0}=1.2, \ell=1$. The first relatively short period of damped oscillations is a kind of memory of the threshold BH state $r_{0}=2 M$, and it goes over into the characteristic quasinormal mode of the wormhole at later times.

TABLE I. Quasinormal mode frequencies $\omega$ of the electromagnetic field for $\ell=1$ in the CFM metric 15

\begin{tabular}{lcc}
\hline$r_{0}$ & Time-domain & WKB \\
\hline 0 & $0.43751-0.24617 i$ & $0.43782-0.24904 i$ \\
0.2 & $0.45313-0.23549 i$ & $0.45365-0.23682 i$ \\
0.4 & $0.46936-0.22148 i$ & $0.46942-0.22174 i$ \\
0.6 & $0.48557-0.20288 i$ & $0.48509-0.20306 i$ \\
0.75 & $0.49653-0.18495 i$ & $0.49652-0.18498 i$ \\
0.8 & $0.49983-0.17789 i$ & $0.50056-0.17729 i$ \\
1 & $0.50651-0.14686 i$ & $0.50652-0.14733 i$ \\
1.1 & $0.50177-0.11188 i$, echoes & - \\
1.2 & $0.48143-0.06821 i, 0.99460-0.00979 i$ & - \\
1.3 & $0.47713-0.07202 i, 0.56376-0.04650 i$ & - \\
10 & initial outburst, $0.12759-0.03054 i$ & - \\
20 & initial outburst, $0.06552-0.01498 i$ & - \\
\hline
\end{tabular}

cillatory fall-off corresponding to the residual fundamental mode of the boundary $r_{0}=2 M \mathrm{BH}$. Finally, when the parameter $r_{0}$ is further increased, the echoes go over into the established characteristic quasinormal ringing of a wormhole (Fig. 3).

Table I exhibits the dominant quasinormal frequencies of the CFM metric in both regimes. In the $\mathrm{BH}$ case $r_{0} \leq$ $2 M$, the quasinormal modes can be computed with both time-domain integration and WKB methods. Near the threshold, when $r_{0} \gtrsim 2 M$, the WKB formula, implying two turning points, cannot be used since the effective potential has two barriers and four turning points.

We can see that for BHs the results produced by the WKB method and time-domain integration are in a very good concordance, with disagreement always less than one percent. Taking into account that the extraction of frequencies from the profiles obtained with the help of time-domain integration greatly depends on the temporal range which is determined as the "quasinormal ring- ing," a relative error within one percent can be easily ascribed not even to the WKB formula but rather to the arbitrariness of the period of quasinormal ringing. Here we used the 7th order WKB formula with the further Padé approximants such that $\tilde{m}=3$, which gives the best agreement with the results of time-domain integration for the Schwarzschild case $r_{0}=3 /(2 M)$ and also coincides (within a 5-digits accuracy) with the accurate numerical value $0.49652-0.18498 i$. For the case of a wormhole very close to the threshold, we have only a single mode which is the residual or, in a sense, "memory" of the boundary $\mathrm{BH}$ solution. The echoes which follow this residual mode cannot be represented by a single dominant quasinormal mode. Nevertheless, when the parameter $r_{0}$ is further increased, the enveloping oscillations of echoes (visible in Fig.22 align, and the characteristic mode of the wormhole establishes, being reflected in the second frequency given in Table 1 . We can also see that at $r_{0} \gg 2 M$ the real and imaginary parts of the frequency are inversely 
TABLE II. Quasinormal modes of the electromagnetic field for $\ell=1$ for the BK-1 metric 16

\begin{tabular}{lcc}
\hline$r_{0}$ & Time-domain & WKB \\
\hline 1 & $0.53569-0.06300 i$ & $0.53569-0.06299 i$ \\
1.1 & $0.53360-0.06227 i$, echoes & - \\
1.2 & $0.51232-0.05364 i$, echoes & - \\
1.3 & $0.50762-0.04834 i$, echoes & - \\
1.4 & $0.49793-0.04302 i, 0.55835-0.01965 i$ & - \\
1.5 & $0.47806-0.03107 i, 0.55859-0.02937 i$ & - \\
\hline
\end{tabular}
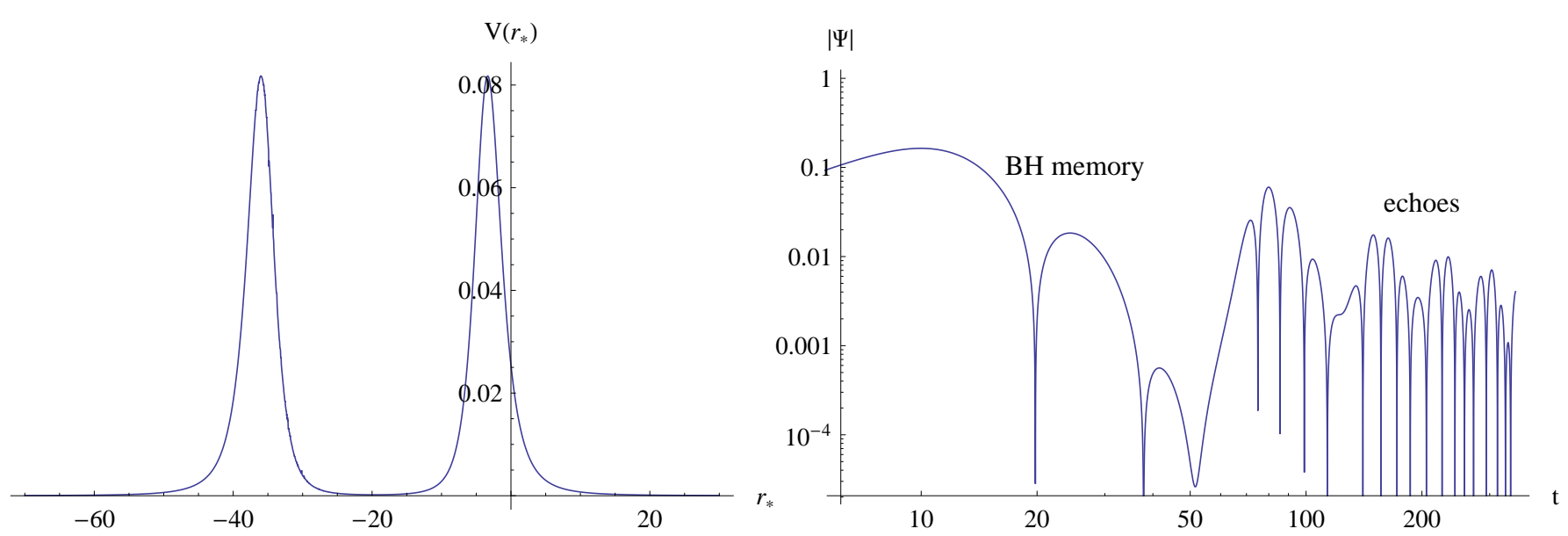

FIG. 4. The effective potential (left) and the logarithmic plot of the time-domain profile (right) for perturbations of the scalar field in the vicinity of the CFM wormhole with $M=1 / 2, r_{0}=1.01, \ell=0$. Several first oscillations (usual for $\ell=0$ perturbations) are indistinguishable from those of the threshold $\mathrm{BH}$ metric (corresponding to $r_{0}=2 M$ ), but at later times they go over into a series of echoes.

proportional to $r_{0}$.

The above description concerned the behavior of electromagnetic perturbations against the background od brane-world BHs and wormholes. It can be verified that massless scalar field perturbations show similar qualitative features, as can be seen in Fig. 4 that depicts the behavior of radial scalar perturbations $(\ell=0)$ of a CFM wormhole.

\section{B. The Bronnikov-Kim-1 (BK-1) metric}

This case represents an atypical example of a $\mathrm{BH}-$ wormhole family of solutions. The metric can be written as

$$
\begin{aligned}
d s^{2}= & \left(1-\frac{2 M}{r}\right)^{2} d t^{2}-\left(1-\frac{r_{0}}{r}\right)^{-1}\left(1-\frac{r_{1}}{r}\right)^{-1} d r^{2} \\
& -r^{2} d \Omega^{2}, \quad r_{1}:=\frac{M r_{0}}{r_{0}-M},
\end{aligned}
$$

The only $\mathrm{BH}$ solution corresponds to the case $r_{0}=$ $r_{1}=2 M$, which coincides with the extremal ReissnerNordstrom metric.

Other values of $r_{0}$ lead either to wormholes (the throat is located at $r=r_{0}$ if $r_{0}>2 M$ or at $r=r_{1}>2 M$ in case $\left.2 M>r_{0}>M\right)$, or to a naked singularity located at $r=2 M$ (if $r_{0}<M$ ) (see more details in Ref. 40]).

Figure 5 shows the picture of evolution of perturbation in the vicinity of the threshold, which is qualitatively the same as for the previous metric, with a distinction related to the length of the initial (residual) quasinormal ringing which is much longer now, and also the frequencies change slower with changing $r_{0}$. This picture simply reflects the fact that larger $r_{0}$ are necessary to deviate from the threshold $\mathrm{BH}$ geometry $r_{0}=2 M$ at the same extent.

\section{The Bronnikov-Kim-2 (BK-2) metric}

This example differs from the previous ones by having a zero value of the Schwarzschild mass of the brane-world $\mathrm{BHs}$ and wormholes. The metric is

$$
\begin{aligned}
& d s^{2}=\left(1-\frac{h^{2}}{r^{2}}\right) d t^{2} \\
& -\left(1-\frac{h^{2}}{r^{2}}\right)^{-1}\left(1+\frac{C-h}{\sqrt{2 r^{2}-h^{2}}}\right)^{-1} d r^{2}-r^{2} d \Omega^{2}
\end{aligned}
$$

The sphere $r=h$ is a simple horizon if $C>0$ and a double horizon if $C=0$. In the case $C<0, B(r)$ has a 

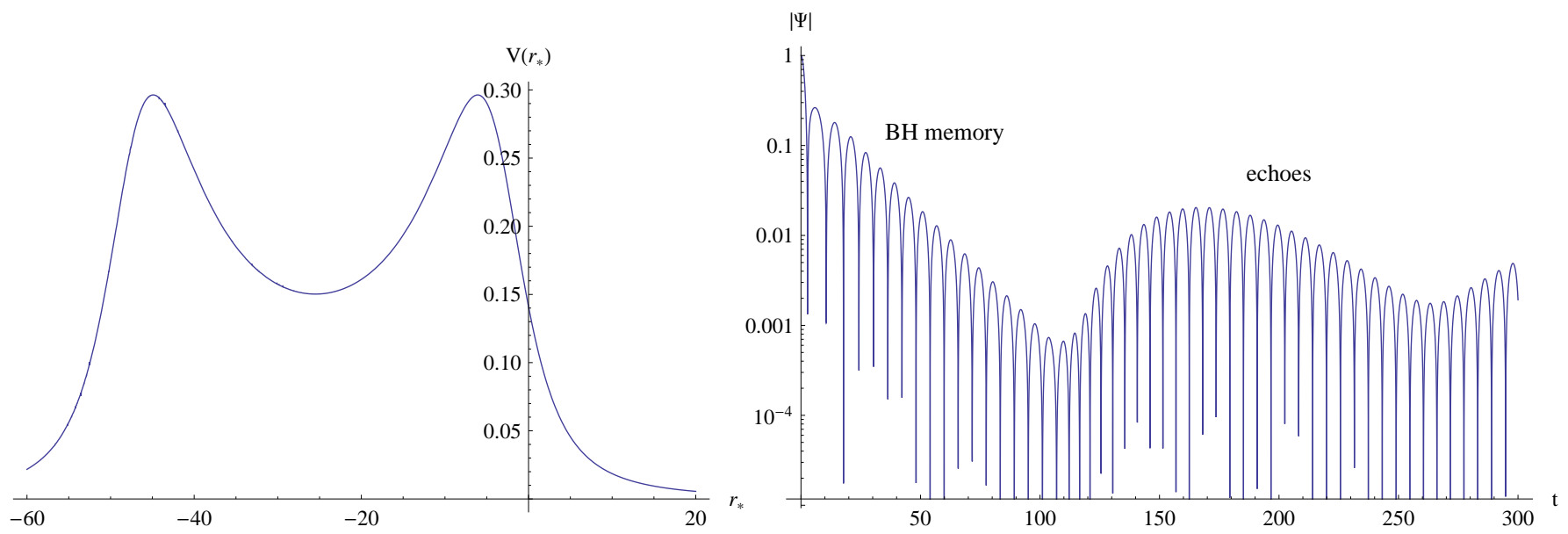

FIG. 5. The effective potential (left) and the semi-logarithmic plot of the time-domain profile (right) for perturbations of the electromagnetic field in the vicinity of the BK-1 wormhole: $M=1 / 2, r_{0}=1.1, \ell=1$. The first long period of damped oscillations is indistinguishable from that of the threshold $\mathrm{BH}$ metric (that corresponds to $r_{0}=2 M$ ), but at later times emerges a series of echoes.
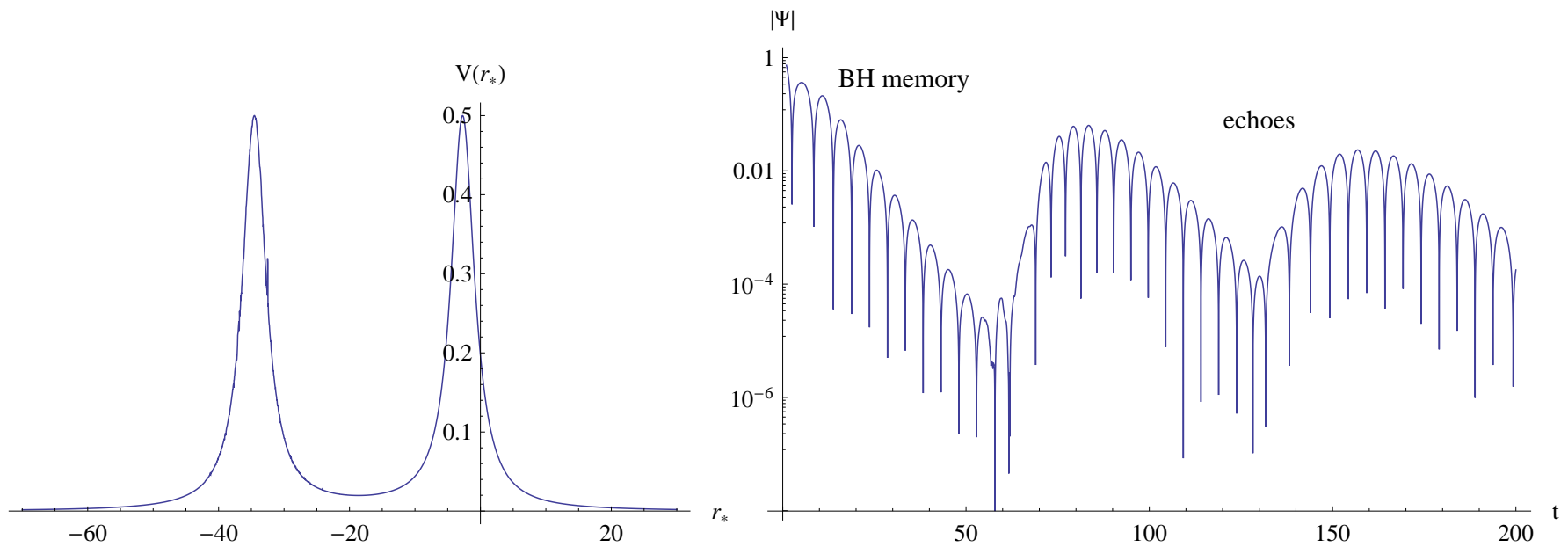

FIG. 6. The effective potential (left) and the semi-logarithmic plot of the time-domain profile (right) for perturbations of the electromagnetic field in the vicinity of the BK-2 wormhole 17 with $M=1 / 2, C=-0.01, \ell=1$. The first long period of damped oscillations is indistinguishable from that of the threshold $\mathrm{BH}$ metric (corresponding to $C=0$ ), but at later times it goes over into a series of echoes.

TABLE III. Quasinormal modes of the electromagnetic field for $\ell=1$ in the BK-2 metric 17.

\begin{tabular}{lcc}
\hline$C$ & Time-domain & WKB \\
\hline 1.5 & $0.53695-0.34301 i$ & $0.54096-0.33905 i$ \\
1 & $0.57668-0.31754 i$ & $0.57663-0.31785 i$ \\
0.5 & $0.61726-0.27547 i$ & $0.61983-0.27457 i$ \\
0.1 & $0.64156-0.22399 i$ & $0.64144-0.22379 i$ \\
0 & $0.64439-0.20921 i$ & $0.64435-0.20900 i$ \\
-0.01 & $0.64306-0.20529 i$, echoes & - \\
-0.1 & $0.61509-0.09685 i$, echoes & - \\
-0.3 & $0.59897-0.07043 i, 0.70323-0.03991 i$ & - \\
-0.5 & initial outburst, $0.73833-0.07600 i$ & - \\
-0.7 & initial outburst, $0.73338-0.09961 i$ & - \\
\hline
\end{tabular}


simple zero at $r=r_{\text {th }}>h$ given by

$$
2 r_{\mathrm{th}}^{2}=h^{2}+(h-C)^{2}
$$

which is a symmetric wormhole throat 40.

The evolution of perturbations in this example is qualitatively similar to the two previous cases: the echoes appears immediately after the threshold, providing wormholes mimicking the $\mathrm{BH}$ behavior. If we consider the quality factor of the mode, which is proportional to the ratio of the real oscillations frequency to the damping rate,

$$
Q \sim \frac{\operatorname{Re}(\omega)}{|\operatorname{Im}(\omega)|}
$$

we can see that oscillations of BHs have a maximum quality factor in the extremal state at the threshold. This is true not only for the last example but for all models under consideration. Moreover, if one considers wormholes with the parameter $C$ (or $r_{0}$ in the previous examples) near the threshold, one can see that the quality factor continues growing and starts to decrease only when echoes are damped. This is also a common feature of all three examples.

\section{CONCLUSIONS}

We have used the higher-order WKB method with Padé approximants [48,52] and time-domain integration 53 in order to analyze the quasinormal ringing of $\mathrm{BH}$ and wormhole solutions in the RS2 brane-world model. The metrics under consideration depend on a continuous parameter interpolating between $\mathrm{BHs}$ and wormholes, so that if the parameter is larger than some threshold value, then the $\mathrm{BH}$ "goes over" into a wormhole. We have shown that this "transition" from BHs to wormholes near the threshold is characterized by echoes: the first stage of damped oscillations, representing the memory of the threshold $\mathrm{BH}$ state is accompanied by a series of echoes at later times. When the same parameter is further increased, the echoes damp and pass on into the characteristic ringing of the wormhole.

This picture is observed for all examples considered here and, apparently, does not depend on the particular model. We believe that this kind of behavior should not depend on the spin of the field as well and should be valid not only for the scalar and electromagnetic fields considered here, but also for the gravitational field because the echoes are induced by appearing of the second symmetric peak in the far left region (in terms of the tortoise coordinate), producing the second scattering and partial reflection of the signal at late times.

Although the characteristic dominant quasinormal frequency of the BHs and wormholes under consideration can behave differently in different examples, there is one feature observed in all the considered models. The ratio of the real oscillation frequency to the damping rate, which is proportional to the quality factor of oscillations, always decreases as the $\mathrm{BH}$ solution moves away from the threshold. On the other hand, the quality factor continues increasing on the other side of the threshold for wormholes which still mimic the $\mathrm{BH}$ behavior, that is, for the residual mode. In other words, wormholes near the threshold are the best oscillators among all the considered examples. Therefore, it is not excluded that this monotonic behavior of the quasinormal frequency might be a more general property of the brane-world models.

\section{ACKNOWLEDGMENTS}

The authors acknowledge the support of the grant 1903950S of Czech Science Foundation $(G A \check{C} R)$. The work of K.B. was partly performed within the framework of the Center FRPP supported by MEPhI Academic Excellence Project (contract No. 02.a03.21.0005, 27.08.2013).

The work was also funded by the RUDN University Program 5-100 and by the RFBR grant No. 19-0200346.
[1] B. P. Abbott et al. [LIGO Scientific and Virgo Collaborations], Phys. Rev. Lett. 116, no. 6, 061102 (2016) arXiv:1602.03837 [gr-qc]].

[2] B. P. Abbott et al. [LIGO Scientific and Virgo Collaborations], Phys. Rev. Lett. 116, no. 22, 221101 (2016) arXiv:1602.03841 [gr-qc]].

[3] C. Goddi et al., Int. J. Mod. Phys. D 26, no. 02, 1730001 (2016) arXiv:1606.08879 [astro-ph.HE]].

[4] K. Akiyama et al. [Event Horizon Telescope Collaboration], Astrophys. J. 875, no. 1, L1 (2019).

[5] T. Damour and S. N. Solodukhin, Phys. Rev. D 76, 024016 (2007); arXiv:0704.2667 [gr-qc]].

[6] R. Konoplya and A. Zhidenko, Phys. Lett. B 756, 350
(2016) |arXiv:1801.03587|[gr-qc]]

[7] R. A. Konoplya, C. Posada, Z. Stuchl?k and A. Zhidenko, Phys. Rev. D 100, no. 4, 044027 (2019) arXiv:1905.08097 [gr-qc]];

[8] C. Posada and C. Chirenti, Class. Quant. Grav. 36, 065004 (2019) arXiv:1811.09589 [gr-qc]];

[9] S. W. Wei and Y. X. Liu, Phys. Rev. D 98, no. 2, 024042 (2018) [arXiv:1803.09530 [gr-qc]]

[10] E. Berti, K. Yagi, H. Yang and N. Yunes, Gen. Rel. Grav. 50, no. 5, 49 (2018) arXiv:1602.04738 [gr-qc]]

[11] V. Cardoso, E. Franzin and P. Pani, Phys. Rev. Lett. 116, no. 17, 171101 (2016) Erratum: [Phys. Rev. Lett. 117, no. 8, 089902 (2016)] arXiv:1602.07309 [gr-qc]]. 
[12] R. A. Konoplya and A. Zhidenko, Rev. Mod. Phys. 83, 793 (2011) arXiv:1102.4014 [gr-qc]].

[13] K. D. Kokkotas and B. G. Schmidt, Living Rev. Rel. 2 (1999) 2 gr-qc/9909058.

[14] E. Berti, V. Cardoso and A. O. Starinets, Class. Quant. Grav. 26, 163001 (2009) arXiv:0905.2975 [gr-qc]].

[15] M. S. Churilova and Z. Stuchlik, arXiv:1911.11823 [grqc].

[16] V. Cardoso, S. Hopper, C. F. B. Macedo, C. Palenzuela and P. Pani, Phys. Rev. D 94, no. 8, 084031 (2016) arXiv:1608.08637 [gr-qc]].

[17] V. Cardoso and P. Pani, Nat. Astron. 1, no. 9, 586 (2017) arXiv:1709.01525 [gr-qc]].

[18] K. W. Tsang et al., Phys. Rev. D 98, no. 2, 024023 (2018) arXiv:1804.04877 [gr-qc]].

[19] R. A. Konoplya, Z. Stuchlik and A. Zhidenko, Phys. Rev. D 99, no. 2, 024007 (2019) arXiv:1810.01295 [gr-qc]].

[20] E. Barausse, V. Cardoso and P. Pani, Phys. Rev. D 89, no. 10, 104059 (2014) arXiv:1404.7149 [gr-qc]].

[21] H. Nakano, N. Sago, H. Tagoshi and T. Tanaka, PTEP 2017, no. 7, 071E01 (2017) arXiv:1704.07175 [gr-qc]].

[22] A. Testa and P. Pani, Phys. Rev. D 98, no. 4, 044018 (2018) arXiv:1806.04253 [gr-qc]].

[23] M. Mirbabayi, arXiv:1807.04843 [gr-qc].

[24] Y. T. Wang, Z. P. Li, J. Zhang, S. Y. Zhou and Y. S. Piao, Eur. Phys. J. C 78, no. 6, 482 (2018) arXiv:1802.02003 $[\mathrm{gr}-\mathrm{qc}]]$.

[25] P. Bueno, P. A. Cano, F. Goelen, T. Hertog and B. Vercnocke, Phys. Rev. D 97, no. 2, 024040 (2018) arXiv:1711.00391 [gr-qc]].

[26] A. Maselli, S. H. Volkel and K. D. Kokkotas, Phys. Rev. D 96, no. 6, 064045 (2017) arXiv:1708.02217 [gr-qc]].

[27] Z. P. Li and Y. S. Piao, Phys. Rev. D 100, no. 4, 044023 (2019) arXiv:1904.05652 [gr-qc]].

[28] Y. T. Wang, J. Zhang, S. Y. Zhou and Y. S. Piao, Eur. Phys. J. C 79, no. 9, 726 (2019) arXiv:1904.00212 [grqc]].

[29] V. Cardoso, V. F. Foit and M. Kleban, JCAP 1908, 006 (2019) doi:10.1088/1475-7516/2019/08/006 arXiv:1902.10164 [hep-th]].

[30] A. Simpson and M. Visser, JCAP 1902, 042 (2019) arXiv:1812.07114 [gr-qc]].

[31] K. A. Bronnikov and J. C. Fabris, Phys. Rev. Lett. 96, 251101 (2006); gr-qc/0511109

[32] K. A. Bronnikov, V. N. Melnikov and H. Dehnen, Gen. Rel. Grav. 39, 973-987 (2007); gr-qc/0611022

[33] S. V. Bolokhov, K. A. Bronnikov, and M. V. Skvortsova, Class. Quantum Grav. 29, 245006 (2012); arXiv: 1208.4619

[34] K. A. Bronnikov, Particles 2018, 1, 5; arXiv: 1802.00098.

[35] J. A. Gonzalez, F. S. Guzman and O. Sarbach, . Class. Quantum Grav. 26, 015010 (2009).

[36] K. A. Bronnikov, J. C. Fabris and A. Zhidenko, Eur. Phys. J. C 71 (11), 1791 (2011); ArXiv: 1109.6576.

[37] K. A. Bronnikov, R. A. Konoplya and A. Zhidenko, Phys. Rev. D 86, 024028 (2012) arXiv:1205.2224 [gr-qc]]
[38] T. Shiromizu, K. Maeda and M. Sasaki, Phys. Rev. D 62 $024012(2000)$

[39] L. Randall and R. Sundrum, Phys. Rev. Lett. 83, 4690 (1999), hep-ph/9906064.

[40] K. A. Bronnikov and S.-W. Kim, Phys. Rev. D 67, 064027 (2003) gr-qc/0212112.

[41] K. A. Bronnikov, V. N. Melnikov and H. Dehnen, Phys. Rev. D 68, 024025 (2003) gr-qc/0304068.

[42] R. Casadio, A. Fabbri and L. Mazzacurati, Phys. Rev. D 65, 084040 (2001); gr-qc/0111072.

[43] C. Germani and R. Maartens, Phys. Rev. D 64, 124010 (2001); hep-th/0107011

[44] E. Abdalla, B. Cuadros-Melgar, A. B. Pavan and C. Molina, Nucl. Phys. B 752, 40 (2006) gr-qc/0604033.

[45] R. A. Konoplya and C. Molina, Phys. Rev. D 71, 124009 (2005) gr-qc/0504139.

[46] R. A. Konoplya and A. Zhidenko, JCAP 1612, 043 (2016) arXiv:1606.00517 [gr-qc]].

[47] R. A. Konoplya and A. Zhidenko, Phys. Rev. D 81, 124036 (2010) arXiv:1004.1284 [hep-th]].

[48] B. F. Schutz and C. M. Will, Astrophys. J. 291, L33 (1985).

[49] S. Iyer and C. M. Will, Phys. Rev. D 35, 3621 (1987).

[50] J. Matyjasek and M. Opala, Phys. Rev. D 96, no. 2, 024011 (2017) arXiv:1704.00361 [gr-qc]]

[51] R. A. Konoplya, A. Zhidenko and A. F. Zinhailo, Class. Quant. Grav. 36, 155002 (2019) arXiv:1904.10333 [gr$\mathrm{qc}]]$.

[52] R. A. Konoplya, Phys. Rev. D 68, 024018 (2003) |grqc/0303052]

[53] C. Gundlach, R. H. Price and J. Pullin, Phys. Rev. D 49, 883 (1994) gr-qc/9307009.

[54] A. F. Zinhailo, Eur. Phys. J. C 78, no. 12, 992 (2018) arXiv:1809.03913 [gr-qc]].

[55] P. D. Roy, S. Aneesh and S. Kar, arXiv:1910.08746 [grqc].

[56] A. Ovgun, I. Sakalli and H. Mutuk, arXiv:1904.09509 [gr-qc].

[57] R. Oliveira, D. M. Dantas, V. Santos and C. A. S. Almeida, Class. Quant. Grav. 36, no. 10, 105013 (2019) arXiv:1812.01798 [gr-qc]].

[58] J. L. Bl?zquez-Salcedo, X. Y. Chew and J. Kunz, Phys. Rev. D 98, no. 4, 044035 (2018) arXiv:1806.03282 [gr$\mathrm{qc}]]$.

[59] R. A. Konoplya, Phys. Lett. B 784, 43 (2018) arXiv:1805.04718 [gr-qc]].

[60] S. Aneesh, S. Bose and S. Kar, Phys. Rev. D 97, no. 12, 124004 (2018) arXiv:1803.10204 [gr-qc]].

[61] E. Abdalla, C. B. M. H. Chirenti and A. Saa, Phys. Rev. D 74, 084029 (2006) doi:10.1103/PhysRevD.74.084029 gr-qc/0609036.

[62] M. S. Churilova, R. A. Konoplya and A. Zhidenko, arXiv:1911.05246 [gr-qc].

[63] M. A. Cuyubamba, R. A. Konoplya and A. Zhidenko, Phys. Rev. D 98, no. 4, 044040 (2018) arXiv:1804.11170 [gr-qc]]. 